\title{
Pós-Graduação em Cardiologia: o Papel da Linha de Pesquisa em Hemodinâmica e Cardiologia Intervencionista na Produção de Conhecimento e na Qualificação de Profissionais no Sul do País
}

\author{
Lucia Campos Pellanda', Rogério Sarmento-Leite' ${ }^{1}$ Fernanda Poester ${ }^{1}$, Ângelo de Souza' \\ Beatriz D. Schaan', Alexandre Schaan de Quadros', Carlos A. M. Gottschall'
}

\section{RESUMO}

Introdução: Os programas de pós-graduação estão evoluindo no Brasil, com a implementação e o aperfeiçoamento de um sistema de avaliação que estimula a produção científica e sua reestruturação. Objetivo: Identificar o impacto da linha de pesquisa em Angioplastia e Implantes de Stents Coronarianos do Programa de Pós-Graduação em Ciências da Saúde/Cardiologia da Fundação Universitária de Cardiologia, em relação à proporção de trabalhos publicados, tempo até a formação e destino dos egressos. Método: Analisadas as dissertações e teses defendidas entre julho de 2002 e janeiro de 2009, pertencentes às linhas de pesquisa relacionadas à Hemodinâmica e Cardiologia Intervencionista e suas publicações. A atual situação dos egressos em relação a atividades assistenciais e ligadas a docência e/ou atividades de pesquisa também foram levantadas. Os dados foram analisados no programa SPSS, sendo as variáveis categóricas descritas em forma de proporções e as contínuas, de médias e desvios padrão. Resultados: Dos 91 trabalhos dos programas de pós-graduação, 71 (78,02\%) eram dissertações e $20(21,9 \%)$, teses. A linha de pesquisa em Angioplastia e Implantes de Stents Coronarianos foi responsável por $18,3 \%(13 / 71)$ das dissertações e por $30 \%$ (6/20) das teses. A maioria dos alunos era constituída por médicos $(9 / 13$ ou $69 \%)$ e do sexo masculino (8/13 ou $61 \%)$. Com tempo médio de 8 meses entre a defesa e a publicação, geraram 5 artigos em revistas internacionais e 9 em periódicos nacionais. Todos os egressos de programas de pós-graduação exercem atividades de docência e/ou assistenciais na instituição de origem, em outras instituições dentro e fora do Rio Grande do Sul ou no exterior. Conclusões: Grande parte dos trabalhos gerados no Programa de Pós-Graduação em Ciências da Saúde/Cardiologia da Fundação Universitária de Cardiologia foi publicada. A

\section{ABSTRACT}

Graduate Studies in Cardiology: the Role of the Research Line in Hemodynamics and Interventional Cardiology in Producing Knowledge and in Professional Qualification in Southern Brazil

Introduction: Graduation programs are evolving in Brazil with the implementation and improvement of an assessment system which encourages scientific production and restructuring. Objective: Identify the impact of the Angioplasty and Coronary stenting research line of the graduation program in Health Sciences/Cardiology, Cardiology University Foundation, regarding the number of papers published, time to conclusion and destiny of graduates. Method: Dissertations and theses presented from July 2002 to January 2009 in the area of Hemodynamics and Interventional Cardiology and their publications were analyzed. The current status of medical, teaching and research activities of these graduates was also investigated. Data were analyzed by the SPSS program and categorical variables are described in ratios and continuous variables are described as mean and standard deviation. Results: Of the 91 studies in the graduate programs, $71(78.02 \%)$ were dissertations and $20(21.9 \%)$ were theses. The Angioplasty and Coronary Stenting research line was responsible for $18.3 \%(13 / 71)$ of the dissertations and for $30 \%(6 / 20)$ of the theses. Most of the graduate students were physicians $(9 / 13$ or $69 \%$ ) and male (8/13 or $61 \%)$. With a mean time of 8 months from dissertation to publication, 5 papers were published in international journals and 9 in national journals. All of the graduates have medical or teaching activities at their institution of origin, in other institutions in and out of the State of Rio Grande do Sul and abroad. Conclusions: A large part of the papers produced in the graduation programs of the Cardiology University

1 Instituto de Cardiologia do Rio Grande do Sul/Fundação Universitária de Cardiologia - Porto Alegre, RS, Brasil.

Correspondência: Rogério Sarmento-Leite. Av. Princesa Isabel, 395 - Setor de Hemodinâmica - Santana - Porto Alegre, RS, Brasil CEP 90620-001

E-mail: sarmentoleite@terra.com.br

Recebido em: 9/4/2009 • Aceito em: 21/6/2009 
Pellanda LC, et al. Pós-Graduação em Cardiologia: o Papel da Linha de Pesquisa em Hemodinâmica e Cardiologia Intervencionista na Produção de Conhecimento e na Qualificação de Profissionais no Sul do País. Rev Bras Cardiol Invas. 2009;17(2):239-45.

maioria dos egressos do programa inseriu-se em atividades de docência em diferentes serviços e instituições.

Descritores: Cardiologia/educação. Ensino superior. Qualificação profissional. Dissertações acadêmicas como assunto. Educação de pós-graduação em Medicina.

A pós-graduação tem passado, recentemente, por uma enorme evolução no Brasil, com a implementação e o aperfeiçoamento de um sistema de avaliação que estimulou a produção científica e a reestruturação dos programas. A área da Medicina é uma das que apresentaram mudanças mais significativas, com importante incremento da produção científica de nível internacional, chegando inclusive a se equiparar a áreas tradicionalmente mais produtivas, como a Física e a Química.

Dentro da Medicina, a Cardiologia é uma das especialidades em que houve grande avanço no desenvolvimento de metodologias de pesquisa e análise, cada vez mais complexas, para avaliar a efetividade de intervenções preventivas e terapêuticas e utilizar as evidências científicas obtidas para guiar a prática clínica.

O Programa de Pós-Graduação em Ciências da Saúde/ Cardiologia da Fundação Universitária de Cardiologia foi criado em 1998 e reestruturado em 2002, de acordo com as novas diretrizes da Coordenação de Aperfeiçoamento de Pessoal de Nível Superior (CAPES). Nessa trajetória, sempre demonstrou compromisso com o desenvolvimento qualitativo da pesquisa e da docência, em um contexto marcado pela inovação tecnológica e pelo avanço da ciência, procurando áreas de atuação que representem a oportunidade de firmar a posição do autor brasileiro na área das Ciências da Saúde, por meio da produção de conhecimento gerado em nosso meio.

O objetivo fundamental do Programa de Pós-Graduação em Ciências da Saúde é a formação de recursos humanos qualificados para o exercício das atividades de pesquisa, desenvolvimento científico e de ensino, culminando com a obtenção dos graus de Mestre e de Doutor, de acordo com a legislação em vigor. O Mestrado tem por objetivo aprofundar o conhecimento acadêmico de profissionais da área da Saúde, bem como possibilitar o desenvolvimento da habilidade para executar pesquisa em um campo específico, sob orientação. O Doutorado, por sua vez, tem por objetivo habilitar o profissional da área da Saúde para, de forma independente, realizar, dirigir e orientar investigação, buscando o avanço e a geração de conhecimentos.

O grande desenvolvimento da Cardiologia permitiu a evolução de linhas de pesquisa específicas, entre
Foundation were published. Most of the graduates in the program have teaching activities in different services and institutions.

Descriptors: Cardiology/education. Education, higher. Credentialing. Dissertations, academic as topic. Education, medical, graduate.

elas as relacionadas à Hemodinâmica e Cardiologia Intervencionista. Na Pós-Graduação em Ciências da Saúde/Cardiologia da Fundação Universitária de Cardiologia, a linha de pesquisa em Angioplastia e Implantes de Stents Coronarianos busca avaliar criticamente resultados obtidos com intervenção coronariana percutânea, especialmente quanto à ocorrência de complicações e sua detecção, a partir de fatores de risco pré, trans e pós-procedimento, tendo como meta sua prevenção.

Inserido no contexto de constante autoavaliação para o aprofundamento das mudanças nos cursos de pós-graduação do País, o presente trabalho teve por objetivo identificar as defesas da linha de pesquisa em Angioplastia e Implantes de Stents Coronarianos do Programa de Pós-Graduação em Ciências da Saúde/ Cardiologia da Fundação Universitária de Cardiologia, identificando suas particularidades em relação à proporção de trabalhos publicados, ao tempo até a formação, e ao destino dos egressos.

\section{MÉTODO}

Foram analisadas todas as dissertações e teses defendidas no âmbito do Programa de Pós-Graduação em Ciências da Saúde/Cardiologia da Fundação Universitária de Cardiologia a partir de julho de 2002, quando esse programa, seguindo normas da CAPES, foi ampla e profundamente reestruturado, até janeiro de 2009, e selecionadas aquelas pertencentes às linhas de pesquisa relacionadas à Hemodinâmica e Cardiologia Intervencionista.

Foram buscadas no PubMed, na Scientific Electronic Library Online (SciELO) e em páginas eletrônicas da Revista Brasileira de Cardiologia Invasiva as publicações relacionadas a essas teses e dissertações. Quando não foi possível encontrar a publicação, os egressos foram contatados.

Adicionalmente, foram buscadas informações sobre a atual situação dos egressos em relação a atividades assistenciais e ligadas à docência e/ou atividades de pesquisa, por meio da Plataforma Lattes, do Conselho Nacional de Desenvolvimento Científico e Tecnológico (CNPq).

Todos os resultados foram tabulados e analisados com auxílio do programa SPSS versão 12.0 para Windows. As variáveis categóricas estão descritas sob 
a forma de proporções e as variáveis contínuas, sob a forma de médias e desvios padrão.

\section{RESULTADOS}

Durante o período de julho de 2002 a janeiro de 2009, foram concluídos 91 trabalhos no Programa de Pós-Graduação em Ciências da Saúde/Cardiologia da Fundação Universitária de Cardiologia, dos quais 71 $(78,02 \%)$ eram dissertações de mestrado e 20 (21,9\%) eram teses de doutorado (Tabela 1). Os números de defesas por ano de mestrado e doutorado podem ser observados na Figura 1.

A linha de pesquisa em Angioplastia e Implantes de Stents Coronarianos é orientada por três professores.
Para o presente trabalho, é relatada somente a produtividade de dois orientadores, já que o terceiro teve ingresso recente no programa. Treze dissertações de mestrado e seis teses de doutorado pertencentes a essa linha de pesquisa correspondem a 18,3\% das dissertações e 30\% das defesas da Pós-Graduação em Ciências da Saúde/Cardiologia da Fundação Universitária de Cardiologia. Considerando a entrada inicial no programa, os alunos do curso de pós-graduação, nessa área específica de atuação, eram na maioria médicos (9/13 ou $69 \%$ ) e do sexo masculino (8/13 ou $61 \%$ ). Constatamos que aqueles que levaram menos tempo entre o término da graduação e o início da pós-graduação foram os que mais cedo concluíram e apresentaram as dissertações ou teses.

TABELA 1

Comparação da produção científica da linha de pesquisa em Hemodinâmica e Cardiologia Intervencionista com as demais linhas do programa de pós-graduação

\begin{tabular}{|c|c|c|c|c|c|c|c|}
\hline \multirow[t]{2}{*}{ Linha de pesquisa } & \multicolumn{3}{|c|}{ Defesas } & \multicolumn{3}{|c|}{ Publicações } & \multirow{2}{*}{$\begin{array}{l}\text { Tempo médio } \\
\text { até a publicação }\end{array}$} \\
\hline & DO & ME & Total & Nac & Int & Total & \\
\hline $\begin{array}{l}\text { Angioplastia e Implantes } \\
\text { de Stents Coronarianos }\end{array}$ & 6 & 13 & 19 & 6 & 8 & 14 & 8 meses \\
\hline Aterogênese e Trombose & - & 6 & 6 & - & 3 & 3 & 2 anos e 3 meses \\
\hline $\begin{array}{l}\text { Estudos dos Fatores de Risco } \\
\text { para Doenças Cardiovasculares }\end{array}$ & - & 6 & 6 & 1 & 1 & 2 & 2 anos \\
\hline $\begin{array}{l}\text { Avanços Propedêuticos e } \\
\text { Terapêuticos no Estudo das } \\
\text { Cardiopatias Estruturais e }\end{array}$ & & & & & & & \\
\hline Funcionais do Período Pré-Natal & 3 & 8 & 11 & 4 & 3 & 7 & 1 ano e 4 meses \\
\hline $\begin{array}{l}\text { Estudo da Função Diastólica } \\
\text { Ventricular do Feto }\end{array}$ & 3 & 1 & 4 & 3 & 2 & 5 & 2 anos e 5 meses \\
\hline $\begin{array}{l}\text { Fisiopatologia e Avaliação das } \\
\text { Cardiopatias Congênitas e } \\
\text { Adquiridas na Infância }\end{array}$ & - & - & - & - & - & - & - \\
\hline $\begin{array}{l}\text { Avaliação Anatomofuncional } \\
\text { da Terapêutica Cirúrgica }\end{array}$ & - & 9 & 9 & 8 & 1 & 9 & 2 anos \\
\hline $\begin{array}{l}\text { Avanços em Métodos de } \\
\text { Diagnóstico Cardiológico }\end{array}$ & 3 & 13 & 16 & 11 & - & 11 & 2 anos \\
\hline $\begin{array}{l}\text { Estudos Funcionais e } \\
\text { Intervenções Experimentais }\end{array}$ & 1 & 5 & 6 & 3 & 1 & 4 & 1 ano \\
\hline $\begin{array}{l}\text { Estudos Avançados em } \\
\text { Fibrilação Atrial }\end{array}$ & 2 & 10 & 12 & 7 & 1 & 8 & 6 meses \\
\hline $\begin{array}{l}\text { Biologia Molecular de Doenças } \\
\text { Crônico-Degenerativas com Ênfase } \\
\text { em Doenças Cardiovasculares; } \\
\text { Terapia Gênica e Celular em }\end{array}$ & & & & & & & \\
\hline $\begin{array}{l}\text { Doenças Cardíacas } \\
\text { Reabilitação e Educação }\end{array}$ & - & - & - & - & - & - & - \\
\hline Transdisciplinar em Cardiologia & 2 & - & 2 & 1 & - & 1 & 1 ano \\
\hline Total & 20 & 71 & 91 & 44 & 20 & 64 & - \\
\hline
\end{tabular}



na Produção de Conhecimento e na Qualificação de Profissionais no Sul do País. Rev Bras Cardiol Invas. 2009;17(2):239-45.

O tempo desde a defesa até a publicação pode ser observado na Tabela 1. Em relação ao total de 91 defesas do programa de pós-graduação de 2002 a 2009, o total de publicações nesse período foi de 64, o que origina uma média de 9,1 publicações por ano. A participação da linha de pesquisa em Angioplastia e Implantes de Stents Coronarianos em relação às demais, em termos de trabalhos concluídos e publicados, pode ser observada na Figura 2.

As dissertações e teses dessa linha de pesquisa geraram 5 publicações plenas em revistas internacionais e 9 em revistas nacionais, com tempo médio de 8 meses desde a defesa até a publicação dos resultados (Quadro 1). Três das dissertações defendidas em 2008 e uma defendida em janeiro de 2009 foram submetidas para publicação, mas ainda aguardam parecer dos revisores.

Com relação ao destino dos egressos, um encontra-se atualmente exercendo atividades assistenciais e

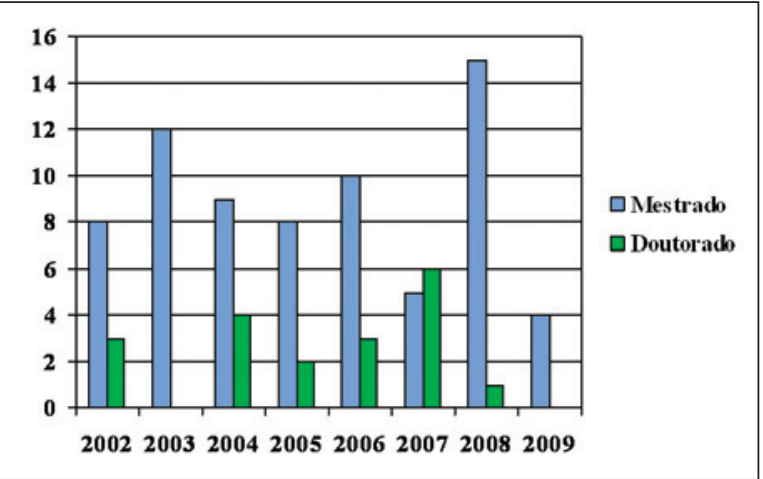

Figura 1 - Número de defesas de mestrado e doutorado no Programa de Pós-Graduação em Ciências da Saúde/Cardiologia da Fundação Universitária de Cardiologia por ano, de 2002 a 2009.

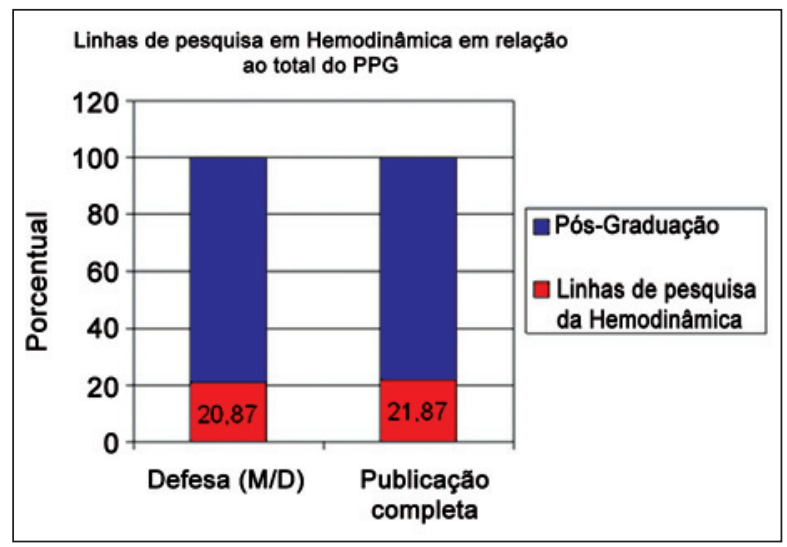

Figura 2 - Comparação da linha de pesquisa em Angioplastia e Implantes de Stents Coronarianos em relação à produção do Programa de Pós-Graduação em Ciências da Saúde/Cardiologia da Fundação Universitária de Cardiologia. $\mathrm{D}=$ doutorado; $\mathrm{M}=$ mestrado; PPG = programa de pós-graduação. de pesquisa e docência no exterior, cinco exercem atividades clínicas e de docência em outros Centros de Hemodinâmica estabelecidos no interior do Estado do Rio Grande do Sul, dois têm o mesmo perfil de atuação em outras unidades da Federação, três permanecem no Serviço de Hemodinâmica da instituição, exercendo atividades de pesquisa e preceptoria, e dois desempenham atividades exclusivamente assistenciais.

\section{DISCUSSÃO}

Embora limitada por ser área de atuação da Cardiologia e com pequeno número de orientadores, a linha de pesquisa em Angioplastia e Implante de Stents Coronarianos do Programa de Pós-Graduação em Ciências da Saúde/Cardiologia da Fundação Universitária de Cardiologia evidencia grande potencial produtivo. Nesse sentido, é fundamental que esforços sejam canalizados para aprimorar, diversificar e multiplicar as pesquisas, ampliando o leque de opções dos veículos de publicação, o que promoverá maior inserção do programa e suas linhas de pesquisa e crescente aumento na qualidade das publicações, que inevitavelmente culminará com o alcance de periódicos de maior repercussão e maior fator de impacto.

Isso, aliás, é uma determinação da CAPES, que atribui peso significativo na nota das avaliações dos programas de pós-graduação no que se refere ao impacto do periódico em que o trabalho foi publicado. Para o triênio de 2004 a 2007, a CAPES considerou que a produção científica dos programas da área de Medicina I deveria representar $30 \%$ da nota total. Nesse quesito, 50\% compreendiam o número de publicações qualificadas do programa por docente permanente; $40 \%$, a distribuição das publicações qualificadas em relação ao corpo docente; e $10 \%$, outras produções consideradas relevantes ${ }^{2}$. Para o próximo triênio, a CAPES sugeriu aumentar o peso da produção científica para $40 \%$, além de propor uma nova classificação Qualis para os periódicos. De acordo com a nova classificação, com oito estratos, os dois estratos superiores (A1 e A2) incluem os periódicos de mais alta qualidade, nos quais a área julga que deva sinalizar para avançar a qualidade da produção científica de sua comunidade. Pretende-se, assim, que o Qualis tenha também um papel indutor, não se limitando a analisar os locais de publicação, mas indicando também onde se deve publicar ${ }^{3}$.

O tempo desde a formatura até a divulgação final dos resultados científicos da pós-graduação também precisa ser encurtado e, conforme sugere a CAPES, a versão final deveria ser enviada o mais breve possível para publicação, idealmente antes mesmo da defesa. Nessa linha, o Programa de Pós-Graduação em Ciências da Saúde/Cardiologia da Fundação Universitária de Cardiologia já solicita a seus alunos que a dissertação ou tese seja apresentada na forma de um artigo original por ocasião de sua defesa pública. 


\section{QUADRO 1}

Publicações da linha de pesquisa em Hemodinâmica e Cardiologia Intervencionista

\begin{tabular}{|c|c|c|c|c|c|c|}
\hline Orientador & $\begin{array}{c}\text { Aluno de } \\
\text { pós-graduação }\end{array}$ & Graduação & Nível & $\begin{array}{l}\text { Ano de } \\
\text { defesa }\end{array}$ & $\begin{array}{c}\text { Título da } \\
\text { dissertação/tese }\end{array}$ & Publicação \\
\hline $\begin{array}{l}\text { Carlos } \\
\text { Gottschall }\end{array}$ & $\begin{array}{l}\text { Emerson Carvalho } \\
\text { Perin }\end{array}$ & $\begin{array}{l}\text { Medicina } \\
\text { UFRGS }\end{array}$ & DO & 2002 & $\begin{array}{l}\text { Uso do mapeamento } \\
\text { eletromecânico do ventrículo } \\
\text { esquerdo na avaliação de } \\
\text { viabilidade miocárdica } \\
\text { e determinação da } \\
\text { transmuralidade de um } \\
\text { infarto em pacientes com } \\
\text { doença arterial coronária } \\
\text { estável: validação pela } \\
\text { ressonância nuclear magnética } \\
\text { através da técnica } \\
\text { de realce tardio }\end{array}$ & $\begin{array}{l}\text { Assessing myocardial } \\
\text { viability and infarct } \\
\text { transmurality with left } \\
\text { ventricular } \\
\text { electromechanical } \\
\text { mapping in patients with } \\
\text { stable coronary artery } \\
\text { disease: validation by } \\
\text { delayed-enhancement } \\
\text { magnetic resonance } \\
\text { imaging } \\
\text { Circulation. } \\
\text { 2002;106(8):957-61. }\end{array}$ \\
\hline $\begin{array}{l}\text { Carlos } \\
\text { Gottschall }\end{array}$ & $\begin{array}{l}\text { Valquíria Gálio } \\
\text { Bulcão }\end{array}$ & $\begin{array}{l}\text { Medicina } \\
\text { UPF }\end{array}$ & ME & 2002 & $\begin{array}{c}\text { Pacientes com elevação } \\
\text { enzimática (CPK e CK-MB) } \\
\text { pós-intervenção coronariana } \\
\text { com ou sem implante de stent: } \\
\text { um ano de seguimento }\end{array}$ & $\begin{array}{l}\text { Prognóstico a longo prazo de } \\
\text { pacientes com elevação } \\
\text { enzimática pós intervenção } \\
\text { coronária: angioplastia versus } \\
\text { stent } \\
\text { Rev Bras Cardiol Invas. } \\
\mathbf{2 0 0 8 ; 1 6 ( 2 ) : 2 0 0 - 5 . ~}\end{array}$ \\
\hline $\begin{array}{l}\text { Carlos } \\
\text { Gottschall }\end{array}$ & $\begin{array}{l}\text { Ibsen Suetonio } \\
\text { Trindade }\end{array}$ & $\begin{array}{l}\text { Medicina } \\
\text { UPF }\end{array}$ & DO & 2003 & $\begin{array}{l}\text { Determinação do tempo } \\
\text { mínimo de insuflação } \\
\text { para a completa expansão } \\
\text { e aposição do stent Tenax XR }\end{array}$ & $\begin{array}{l}\text { Determination of the minimum } \\
\text { inflation time necessary for tota } \\
\text { stent expansion and apposition. } \\
\text { an in vitro study } \\
\text { J Invasive Cardiol. } \\
\mathbf{2 0 0 8 ; 2 0 ( 8 ) : 3 9 6 - 8 . ~}\end{array}$ \\
\hline $\begin{array}{l}\text { Carlos } \\
\text { Gottschall }\end{array}$ & $\begin{array}{l}\text { Alexandre Damiani } \\
\text { Azmus }\end{array}$ & $\begin{array}{l}\text { Medicina } \\
\text { UFCSPA }\end{array}$ & DO & 2004 & $\begin{array}{l}\text { Efetividade da } \mathrm{N} \text {-acetilcisteína } \\
\text { na prevenção da insuficiência } \\
\text { renal pós-contraste }\end{array}$ & $\begin{array}{c}\text { Effectiveness of acetylcysteine } \\
\text { in prevention of contrast } \\
\text { nephropathy } \\
\text { J Invasive Cardiol. } \\
\text { 2005;17(2):80-4. } \\
\text { The effect of N-acetylcysteine } \\
\text { in renal function in patients } \\
\text { undergoing cardiac } \\
\text { intervention } \\
\text { Am J Cardiol. } \\
\text { 2002;24:144H. (abstract) }\end{array}$ \\
\hline $\begin{array}{l}\text { Carlos } \\
\text { Gottschall }\end{array}$ & $\begin{array}{c}\text { Alexandre Schaan } \\
\text { deQuadros }\end{array}$ & $\begin{array}{l}\text { Medicina } \\
\text { UFRGS }\end{array}$ & DO & 2004 & $\begin{array}{l}\text { Análise dos resultados a } \\
\text { curto e longo prazo } \\
\text { do implante de } \\
\text { stents coronarianos }\end{array}$ & $\begin{array}{l}\text { Predictive score for target vesse } \\
\text { revascularization after bare meta } \\
\text { coronary stenting } \\
\text { J Invasive Cardiol. } \\
\text { 2006;18(1):22-6. }\end{array}$ \\
\hline $\begin{array}{l}\text { Carlos } \\
\text { Gottschall }\end{array}$ & Iseu Gus & $\begin{array}{l}\text { Medicina } \\
\text { UFRGS }\end{array}$ & DO & 2005 & $\begin{array}{c}\text { Fatores de risco da } \\
\text { doença arterial coronariana } \\
\text { no estado do Rio Grande do Sul: } \\
\text { uma linha de pesquisa }\end{array}$ & $\begin{array}{l}\text { Prevalência dos fatores } \\
\text { de risco da doença arterial } \\
\text { coronariana no estado do } \\
\text { Rio Grande do Sul } \\
\text { Arq Bras Cardiol. } \\
\text { 2002;78(5):478-83. }\end{array}$ \\
\hline
\end{tabular}

$\mathrm{DO}=$ doutorado; Feevale = Centro Universitário Feevale (Novo Hamburgo, RS); FFFCMPA = Fundação Faculdade Federal de Ciências Médicas de Porto Alegre; IPA = Centro Universitário Metodista/Instituto Porto Alegre; ME = mestrado; PUC-RS =Pontifícia Universidade Católica do Rio Grande do Sul; UCPEL = Universidade Católica de Pelotas; UFCSPA = Universidade Federal de Ciências da Saúde de Porto Alegre; UFRGS = Universidade Federal do Rio Grande do Sul; UFSM = Universidade Federal de Santa Maria; UPF = Universidade de Passo Fundo. 
QUADRO 1 (continuação)

Publicações da linha de pesquisa em Hemodinâmica e Cardiologia Intervencionista

\begin{tabular}{|c|c|c|c|c|c|c|}
\hline Orientador & $\begin{array}{c}\text { Aluno de } \\
\text { pós-graduação }\end{array}$ & Graduação & Nível & $\begin{array}{l}\text { Ano de } \\
\text { defesa }\end{array}$ & $\begin{array}{c}\text { Título da } \\
\text { dissertação/tese }\end{array}$ & Publicação \\
\hline $\begin{array}{l}\text { Carlos } \\
\text { Gottschall }\end{array}$ & $\begin{array}{c}\text { Géderson } \\
\text { Rossato }\end{array}$ & $\begin{array}{l}\text { Medicina } \\
\text { FFFCMPA }\end{array}$ & ME & 2005 & $\begin{array}{c}\text { Estudo de complicações } \\
\text { imediatas do cateterismo } \\
\text { cardíaco diagnóstico } \\
\text { em adultos }\end{array}$ & $\begin{array}{l}\text { Análise das complicações } \\
\text { relacionadas ao } \\
\text { cateterismo cardíaco } \\
\text { Rev Bras Cardiol Invas. } \\
\text { 2007; 15(1):44-51. }\end{array}$ \\
\hline $\begin{array}{l}\text { Rogério } \\
\text { Sarmento- } \\
\text { Leite/ } \\
\text { Alexandre } \\
\text { Schaan de } \\
\text { Quadros }\end{array}$ & $\begin{array}{c}\text { Emiliane Nogueira } \\
\text { de Souza }\end{array}$ & $\begin{array}{c}\text { Enfermagem } \\
\text { UFSM }\end{array}$ & ME & 2005 & $\begin{array}{c}\text { Preditores de qualidade } \\
\text { de vida em pacientes } \\
\text { com síndrome coronariana } \\
\text { aguda com } \\
\text { supradesnivelamento } \\
\text { de ST após } 6 \text { meses } \\
\text { de internação hospitalar }\end{array}$ & $\begin{array}{l}\text { Preditores de mudança na } \\
\text { qualidade de vida após } \\
\text { um evento coronariano } \\
\text { agudo } \\
\text { Arq Bras Cardiol. } \\
\text { 2008;91(4):252-9. }\end{array}$ \\
\hline $\begin{array}{l}\text { Carlos } \\
\text { Gottschall }\end{array}$ & $\begin{array}{l}\text { JoséCarlos } \\
\text { Jotz }\end{array}$ & $\begin{array}{l}\text { Medicina } \\
\text { UFRGS }\end{array}$ & ME & 2006 & $\begin{array}{l}\text { Verificação do efeito do } \\
\text { Chelidonium majus D3 } \\
\text { sobre a hipercolesterolemia } \\
\text { experimentalmente induzida } \\
\text { em coelhos }\end{array}$ & $\begin{array}{c}\text { Efeito do } \\
\text { Chelidonium majus D3 } \\
\text { sobre a hipercolesterolemia } \\
\text { experimentalmente } \\
\text { induzida em coelhos } \\
\text { Rev AMRIGS. } \\
\mathbf{2 0 0 8 ; 5 2 ( 1 ) : 2 9 - 3 3 . ~}\end{array}$ \\
\hline $\begin{array}{l}\text { Rogério } \\
\text { Sarmento- } \\
\text { Leite }\end{array}$ & $\begin{array}{c}\text { Cristiano de Oliveira } \\
\text { Cardoso }\end{array}$ & $\begin{array}{l}\text { Medicina } \\
\text { UCPEL }\end{array}$ & ME & 2006 & $\begin{array}{l}\text { Efetividade da prótese de } \\
\text { Amplatzer para fechamento } \\
\text { percutâneo do defeito } \\
\text { do septo interatrial } \\
\text { tipo ostium secundum }\end{array}$ & $\begin{array}{l}\text { Efetividade da prótese de } \\
\text { Amplatzer para fechamento } \\
\text { percutâneo do defeito } \\
\text { do septo interatrial } \\
\text { tipo ostium secundum } \\
\text { Arq Bras Cardiol. } \\
\text { 2007;88(4):384-9. } \\
\text { Transcatheter closure of } \\
\text { atrial septal defect with } \\
\text { Amplatzer device in } \\
\text { children aged less than } \\
\text { 10 years old: immediate } \\
\text { and late follow-up } \\
\text { Catheter Cardiovasc Interv. } \\
\text { 2008;71(2):231-6. }\end{array}$ \\
\hline $\begin{array}{l}\text { Carlos } \\
\text { Gottschall }\end{array}$ & $\begin{array}{l}\text { Conceição } \\
\text { Lemos }\end{array}$ & $\begin{array}{l}\text { Psicologia } \\
\text { PUC-RS }\end{array}$ & ME & 2006 & $\begin{array}{l}\text { Associação entre depressão, } \\
\text { ansiedade e qualidade } \\
\text { de vida em pacientes que } \\
\text { apresentam quadro de } \\
\text { pós-infarto do miocárdio }\end{array}$ & $\begin{array}{l}\text { Associação entre depressão, } \\
\text { ansiedade e qualidade } \\
\text { de vida em pacientes que } \\
\text { apresentam quadro de } \\
\text { pós-infarto do miocárdio } \\
\text { Psicologia: Teoria e Pesquisa. } \\
\text { 2008;24(4):471-6. }\end{array}$ \\
\hline $\begin{array}{l}\text { Rogério } \\
\text { Sarmento- } \\
\text { Leite }\end{array}$ & $\begin{array}{l}\text { Ana Cristina } \\
\text { Sudbrack }\end{array}$ & $\begin{array}{c}\text { Fisioterapia } \\
\text { IPA }\end{array}$ & ME & 2007 & $\begin{array}{l}\text { Efetividade do exercício } \\
\text { físico na reabilitação de } \\
\text { pacientes com claudicação } \\
\text { intermitente }\end{array}$ & $\begin{array}{l}\text { Efetividade do exercício } \\
\text { na claudicação } \\
\text { Rev Bras Cardiol Invas. } \\
\text { 2007;15(3):261-6. }\end{array}$ \\
\hline $\begin{array}{l}\text { Rogério } \\
\text { Sarmento- } \\
\text { Leite }\end{array}$ & Carine Ghem & $\begin{array}{l}\text { Biomedicina } \\
\text { Feevale }\end{array}$ & ME & 2008 & $\begin{array}{l}\text { Concentração de bilirrubina } \\
\text { sérica em pacientes com } \\
\text { diagnóstico estabelecido } \\
\text { de doença arterial coronariana }\end{array}$ & $\begin{array}{c}\text { Associação da concentração de } \\
\text { bilirrubina sérica com a carga } \\
\text { aterosclerótica em pacientes com } \\
\text { doença arterial coronariana } \\
\text { Rev Bras Cardiol Invas. } \\
\text { 2008;16(4):445-9. }\end{array}$ \\
\hline
\end{tabular}

DO = doutorado; Feevale = Centro Universitário Feevale (Novo Hamburgo, RS); FFFCMPA = Fundação Faculdade Federal de Ciências Médicas de Porto Alegre; IPA = Centro Universitário Metodista/Instituto Porto Alegre; ME = mestrado; PUC-RS =Pontifícia Universidade Católica do Rio Grande do Sul; UCPEL = Universidade Católica de Pelotas; UFCSPA = Universidade Federal de Ciências da Saúde de Porto Alegre; UFRGS = Universidade Federal do Rio Grande do Sul; UFSM = Universidade Federal de Santa Maria; UPF = Universidade de Passo Fundo. 

na Produção de Conhecimento e na Qualificação de Profissionais no Sul do País. Rev Bras Cardiol Invas. 2009;17(2):239-45.

A autoavaliação permanente e contínua dos programas de pós-graduação em Cardiologia é fundamental para exercermos todo esse potencial de contribuição para com a ciência brasileira, ao identificar possíveis pontos de estrangulamento do sistema, apontar soluções e caminhos para o aperfeiçoamento. Aqui se abre um espaço para uma forte reflexão. Estamos inseridos no modelo da pós-graduação stricto sensu. Nesse contexto, o binômio orientador-aluno é indissociável e fontes financiadoras de pesquisa independentes são imprescindíveis. O crescimento da produção científica só se dá com forte aporte financeiro e grande dedicação dos corpos docente e discente. A CAPES reconhece isso, tanto é que premia aqueles cursos com melhor nota e desempenho conforme rígido sistema de avaliação. Por outro lado, o Brasil e, por conseguinte, o nosso programa estão inseridos em um modelo paradoxal, em que os investimentos na ciência são pequenos e a remuneração é baixa. Isso determina buscas alternativas de financiamento e remuneração. Além disso, pode haver conflito em relação aos objetivos de alguns programas de residência e especialização médica, especialmente aqueles mais estruturados, cujos currículos, agendas e exigências por vezes se sobrepõem aos dos cursos de pós-graduação, fomentando as discussões que envolvem os cursos de mestrado profissionalizante e afastando os doutores da pesquisa básica e de bancada.

A pesquisa só está completa em seus propósitos com a divulgação de seus resultados. A estrutura anterior dos programas de pós-graduação muitas vezes resultava na baixa penetração dos resultados de pesquisa na comunidade científica, com pouca ou nenhuma divulgação internacional dos trabalhos brasileiros. O formato de tese, muitas vezes disponível apenas nas bibliotecas das instituições, impedia sua divulgação mais ampla. No entanto, como apontam Marchini e Caramelli ${ }^{4}$, "a defesa de tese não é o fim de um processo, mas sim seu início, gerando conhecimento a ser divulgado, além de servir de alicerce para novos trabalhos". Fica reforçada, assim, a necessidade de moldarmos os trabalhos às exigências de cada periódico, e isso já se faz na elaboração do projeto de pesquisa. Nessa etapa ainda inicial, que precede até a coleta de dados, os objetivos devem ser muito bem estabelecidos, considerando o potencial de publicação do tema a ser estudado até a língua em que o manuscrito será redigido ${ }^{5-7}$.

Embora limitado por uma amostra ainda pequena, este trabalho pretende destacar a boa potencialidade de geração e difusão de conhecimentos e grande capacidade de nucleação da área de Hemodinâmica e Cardiologia Intervencionista do Programa de Pós-Graduação em Ciências da Saúde/Cardiologia da Fundação Universitária de Cardiologia. Conforme os dados relatados em nosso estudo, grande parte dos trabaIhos realizados nessa área alcança publicação final, e os egressos do programa inserem-se em atividades de docência em diferentes serviços e instituições, muitas vezes estruturados com o auxílio dos mesmos. Uma amostragem de outros programas de nosso País seria interessante para traçarmos um mapa real da contribuição científica e técnica da área em nosso meio.

\section{CONFLITO DE INTERESSES}

Rogério Sarmento-Leite recebeu apoio educacional e de pesquisa das empresas Abbot, Biotronik e Cordis; Bureau de Palestrantes das empresas Biotronik e B-Braun; prestou consultoria técnica para Biotronik; e pertence ao Comitê Científico da Scitech. Alexandre Schaan de Quadros recebeu auxílio para pesquisa das empresas Boston Scientific e Medtronic, além de auxílio para participação em congressos da B-Braun e Medtronic. Os demais autores declararam inexistência de conflito de interesses.

\section{REFERÊNCIAS BIBLIOGRÁFICAS}

1. XXIV Encontro Nacional de Pro-Reitores de Pesquisa e PósGraduação. Ouro Preto, 27-30 de outubro de 2008. [Comunicação pessoal. Prof. Dr. Jorge Guimarães, Presidente da CAPES]

2. CAPES - Critérios de avaliação trienal - 2004-2007. Disponível em: http://www.capes.gov.br/images/stories/download/avaliacao/ CA2007_CienciasSaude.pdf. Acesso em maio 2009.

3. MEC. Coordenação de aperfeiçoamento de pessoal de nível superior - CAPES. Diretoria de Avaliação - DAV. Reestruração do Qualis. Disponível em: http://www.capes.gov.br/images/ stories/download/avaliacao/Restruturacao_Qualis.pdf. Acesso em maio 2009.

4. Marchini JF, Caramelli B. Doutorado em Cardiologia no Instituto do Coração da FMUSP, de 1994 a 2004: Defesa e Publicação. Arq Bras Cardiol. 2008;91(5):315-20.

5. Clausell N, Fuchs FD, Manfroi W, Ribeiro JP. Programa de Pós-graduação em Cardiologia e Ciências Cardiovasculares da Faculdade de Medicina da Universidade Federal do Rio Grande do Sul. Arq Bras Cardiol. 2004;83(6):458-60.

6. Hueb W, Mady C, Ramires JA. Trinta anos de Pós-Graduação em Cardiologia. Arq Bras Cardiol. 2005;85(6):385-7.

7. Kuo KN, Hwang TL, Chen PJ. Physician-scientist: attitude of graduates of clinical medicine graduate schools. J Formos Med Assoc. 2008;107(7):519-26. 